\title{
Trayectorias formativas y la identidad profesional de futuros profesores de matemática
}

\author{
Formative trajectories and the professional identity of pre-service \\ math's teachers
}

\author{
MG. RODRIGO PANES CHAVARRÍA \\ Grupo de Investigación en Educación y Educación Matemática \\ Universidad del Bío-Bio, Chillán, Chile \\ Candidato del Doctorado en Educación en Consorcio, \\ Universidad Católica del Maule, Talca, Chile \\ rpanes@ubiobio.cl
}

\author{
DRA. MARIANA LAZZARO-SALAZAR \\ Doctora en Lingüística \\ Vicerrectoría de Investigación y Postgrado \\ Universidad Católica del Maule, Curicó, Chile \\ mlazzaro@ucm.cl
}

\section{RESUMEN}

Recientemente, la formación inicial docente se ha posicionado, a la luz de las demandas de la sociedad, en una línea de investigación que explora las construcciones identitarias que futuros profesores han desarrollado en el seno de espacios de participación y aprendizaje durante su formación. Como parte de su trayectoria formativa, el futuro profesor no solo convive con tareas y saberes propios de la disciplina, sino que desarrolla relaciones sociales que dan origen a orientaciones identitarias que, posteriormente, incidirán en su práctica profesional. En este contexto, este estudio explora aquellas experiencias y acontecimientos que influyen en las trayectorias formativas y, con ello, en la construcción identitaria de futuros profesores de matemática de una universidad del centro-sur de Chile. Adoptando un diseño biográfico-narrativo, se recolectaron 173 relatos de experiencias formativas mediante una encuesta de caracterización y biogramas donde se observan motivaciones explicativas de la construcción identitaria de 25 profesores en formación. Los resultados muestran que la presencia de un docente referente y sus intervenciones didácticas durante las trayectorias formativas permiten despertar $y$ reafirmar las vocaciones y elecciones para convertirse en profesor de matemática.

Palabras clave: Trayectorias formativas, futuros profesores, identidad, formación inicial docente, biogramas. 


\section{ABSTRACT}

Recently, the area of initial teacher training has been positioned in light of the societal demands within a field of research that explores the identity constructions that preservice teachers have developed within participation and learning spaces during their training. As part of their training trajectory, future teachers not only develop tasks and knowledge of the discipline but also social relationships that help construct identity orientations that will later affect their professional practice. In this context, this study explores those experiences and events that influence the trajectories and, in turn, the identity construction of a group of pre-service math's' teachers from a university in south-central Chile. Adopting a biographical-narrative design, 173 stories of formative experiences were collected through a survey of characterization and through biograms, where the motivations guiding the identity construction of 25 preservice teachers are observed. The results show that the presence of a teacher as a role model and their didactic interventions during the trajectories of these pre-service teachers prompt choices of becoming a professor of mathematics and vocations to be found and reaffirmed.

Key words: Training trajectories, pre-service teachers, identity, initial teacher training, biograms.

\section{INTRODUCCIÓN}

La práctica docente y la orientación pedagógica de un profesor se definen a partir de una serie de acontecimientos, saberes, experiencias, creencias y valores que se desarrollan a lo largo de su formación. Estos periodos de 'construcción' profesional de un docente se conocen comúnmente como trayectorias formativas (Cols, 2008). En este sentido, diversas investigaciones (Sfard y Prusak, 2005; Vaillant, 2010; Vera y Valenzuela, 2012) muestran que los futuros profesores, al reflexionar sobre sus procesos de formación profesional, dan cuenta de una serie de circunstancias, eventos y personajes que van influyendo en su identificación y orientación con respecto a las interrogantes de 'quién quiero ser' y 'para qué' (Evetts, 2003). Así, desde el campo de las ciencias de la educación, se ha visualizado que tanto el contexto sociocultural de formación, como el conjunto de experiencias vividas a lo largo de su formación profesional y la identificación con una disciplina son asociaciones que moldean las trayectorias formativas e influyen, a su vez, de forma fundamental en la construcción de identidad profesional de los profesores (Galaz, 2011). 
Esta construcción de la identidad profesional obedece a múltiples motivaciones y factores socioculturales que influyen e interactúan en el proceso formativo de una persona (Fernández, 2006; Sayago et al., 2008; Avendaño y González, 2012). Como parte de un proceso contextualizado de construcción de la identidad, el estudio de las trayectorias formativas, es decir, el proceso de formación profesional, según lo planteado por Bourdieu (1997), permite el reconocimiento sucesivo de una serie de posicionamientos a los que una misma persona se orienta durante un determinado tiempo y espacio, los cuales se configuran, negocian y transforman a través de las interacciones propias de los procesos de sociabilización. Este posicionamiento dinámico de la identidad conlleva a entender actos, fines y rutinas comunes que se desarrollan en el marco de comunidades de práctica, es decir, un espacio sociocultural en donde los miembros comparten el uso de un repertorio comunicativo disciplinar, formas similares de entender la disciplina y normas sociales que guían su comportamiento (Wenger, 1998; Lazzaro-Salazar, 2013). En este contexto, la construcción de la identidad se puede interpretar a partir de las mismas voces de los actores sociales al reconocer en sus narrativas y discursos los intereses, experiencias, significados e intencionalidades que moldean su identidad profesional y, por consiguiente, su práctica profesional (Bolívar et al., 2005).

La literatura que respalda el desarrollo de esta área de estudio, sin embargo, se ha centrado especialmente en diversos aspectos de las trayectorias formativas y la construcción de la identidad profesional de profesores en ejercicio, desentendiéndose así, en gran medida, del desarrollo de estos procesos de formación docente durante el período de educación formal en pedagogía. En este sentido, el conocer cuáles son los acontecimientos, valores y creencias, entre otros, que marcan la trayectoria formativa y la construcción identitaria de futuros profesores abre la posibilidad de replantear y realizar un acompañamiento de estos procesos desde lo curricular, la visión institucional de disciplina y el quehacer formativo de forma más atingente a las necesidades, expectativas y vivencias de estos futuros profesores (Cols, 2008).

Con el fin entonces de contribuir al desarrollo de este área de investigación con futuros pedagogos, el presente artículo explora los aspectos formativos, es decir, aquellos acontecimientos, actores sociales y experiencias (sobre los cuales nos referiremos aquí como 'motivaciones') que marcan las trayectorias formativas, así como los procesos de construcción de la identidad profesional, tanto en contextos académicos como no académicos de un grupo de futuros profesores de matemáticas de una universidad pública y regional del centro-sur de Chile. 


\section{MARCO TEÓRICO}

\section{Futuros profesores, trayectorias e identidad profesional}

La formación inicial docente durante lasúltimas décadas ha ofrecido a investigadores en educación un amplio campo para el desarrollo de investigación teórica y aplicada, ya sea reconociendo la influencia de la calidad en las instituciones formadoras de profesores en el caso chileno (Ávalos, 2014; Pedraja-Rejas et al., 2012) como en el extranjero (UNESCO, 2013), analizando las iniciativas de políticas educativas aplicables a la formación de profesores (Ávalos, 2014), conociendo modelos de formación de los formadores de profesores (Contreras y Villalobos, 2010), e indagando sobre la calidad de la enseñanza y del aprendizaje y sobre los efectos de la educación universitaria de los futuros profesores (Tatto et al., 2012). Lo que subyace en estos estudios es la atención sobre el futuro profesor, partiendo de la premisa de que el contexto de formación tiene gran influencia en las características de una futura profesión docente. Desde otros constructos integradores, como la identidad, se han podido conocer diversas dimensiones (afectivas, sociales y profesionales) de estos futuros profesores (Vera y Valenzuela, 2007). También, se ha indagado en las decisiones que toman en sus primeras experiencias docentes (Bedacarratx, 2012), y en cómo estos futuros pedagogos se identifican con grupos de profesionales representativos de su futura profesión (Vaillant, 2010). Sin embargo, a pesar de este interés científico (a nivel nacional e internacional) por explorar aquellos aspectos que influyen en la formación profesional de futuros profesores, son escasos los estudios recientes que abordan esta temática desde una perspectiva longitudinal, es decir, que investiguen cómo los acontecimientos, experiencias, creencias, valores e identidades que forman parte de la formación profesional de futuros profesores se desarrollan y re-significan a través del tiempo y de las trayectorias formativas (Cols, 2008).

Desde el campo de las ciencias de la educación, las trayectorias formativas se definen como el conjunto de experiencias y acontecimientos vividos a lo largo de la formación profesional y la identificación con una disciplina que impactan en las decisiones y prácticas profesionales de los futuros profesores (Fernández, 2016). Así entonces, en las trayectorias de formación se gestan los saberes, creencias y valores que construyen las concepciones sobre el futuro quehacer docente y las identidades que luego lo guiarán. Con respecto a esto último, cabe señalar que la identidad profesional es aquí entendida como un aspecto de la identidad social en contextos influidos por la profesión a desempeñar, "una serie de posiciones sucesivamente ocupadas por un mismo agente (o un mismo grupo) en un espacio 
en sí mismo en movimiento y sometido a incesantes transformaciones" (Bourdieu, 1997: 92). Esto incluye, por ejemplo, sus manifestaciones y creencias sobre el contexto universitario, las rutinas de trabajo y aprendizaje, y las apreciaciones hacia el contexto escolar (Sayago et al., 2008). De esta forma, la identidad profesional es expresada en los términos de distinguirse y diferenciarse, desde la cual el '¿qué quiero ser?' y el '¿para qué?' conlleva una condición de identificación y provee garantía a futuro de una adecuación profesional (Sayago et al.,2008). En este contexto, la identidad profesional encierra diversas dimensiones ligadas al campo experiencial, es decir, formas de percibir los problemas y sus posibles soluciones, y al disciplinar, es decir, el compartir conocimientos técnicos comunes a un área de desarrollo profesional (Evetts, 2003), que hacen de las trayectorias formativas procesos dinámicos y cambiantes frente a nuevos acontecimientos y experiencias.

\section{Trayectorias de formación, identidad y entornos formativos}

La construcción de la identidad no es un proceso meramente interno de la persona (ver proyecto de 'ser' de la persona en Habermas, 1989), sino que también forma parte del resultado de una continua negociación de posicionamientos identitarios en contextos sociales (Beijaard et al., 2004). De esta forma, la identidad profesional se constituye en las trayectorias formativas a través de un "mecanismo mediante el cual los profesores se reconocen a sí mismos y son reconocidos por otros como miembros de una determinada categoría social, la categoría de los profesores" (Gysling, 1992:12). En el caso de los futuros profesores de matemática, este mecanismo de reconocimiento es una proyección de su futura profesión ligada a la visión, enseñanza y aprendizaje de la matemática. Esto corresponde con lo planteado por Husu (2007) al considerar que estas identidades profesionales se originan en la práctica verbal y en el intercambio comunicativo con otros al seno de las trayectorias formativas. Así también, la literatura nos da cuenta de ese proceso de sociabilización y de un posicionamiento dinámico de la identidad profesional donde se articulan experiencias, historicidad y situaciones de otredad en ambientes discursivos, contextualizados y de aprendizaje (Navarrete-Cazales, 2015). Esto, además, se puede vincular con la perspectiva sociocultural y situada del aprendizaje (Wenger, 1998; Lave, 1991), la cual sostiene que esta construcción identitaria se produce por la interacción de los miembros en el mismo contexto social donde se desarrollan como futuros profesionales. En este sentido, los aportes de Wenger (2010) proporcionan un marco conceptual a través del cual, en este estudio, se entiende que la identidad profesional de los futuros profesores 
se construye en los procesos de sociabilización al interior de espacios, físicos y sociales, formales e informales, en los que se aprende y se participa, en otras palabras, al interior de determinadas comunidades (Lazzaro-Salazar, 2013; 2017), lo cual, a su vez, constituye las trayectorias formativas de estos futuros profesores (Bolívar et al., 2005).

Con respecto a los avances sobre la comprensión del fenómeno identitario en trayectorias formativas, se puede encontrar que este campo de la investigación de la educación matemática ha permitido aportar al conocimiento de las características del estudiantado (Sfard y Prusak, 2005; Nasir, 2007; Sayago et al., 2008; Cobb et al., 2009). Cabe destacar, sin embargo, que si bien estos estudios avanzan en caracterizar los acontecimientos cronológicos que influyen sobre la formación identitaria de futuros profesores, se ha avanzado poco, especialmente si tenemos en cuenta el contexto chileno, en la profundización de aspectos tales como el rol de los actores sociales y las experiencias pedagógicas en la construcción de identidad en el contexto de las trayectorias formativas de estos estudiantes. En este sentido, estudios de trayectorias del profesor en servicio o la identidad de los formadores caracterizan más comúnmente esta área de investigación (Husu, 2007; Grootenboer, 2006). Por ello, la búsqueda de esta profundización del conocimiento sobre los procesos constitutivos de las trayectorias formativas de futuros profesores es la que motiva este estudio.

De esta forma, al explorar las trayectorias formativas de un grupo de futuros profesores, el presente artículo se centra en aquellos acontecimientos y experiencias fuertemente vinculados con el rol vital que cumple el docente de matemáticas en las elecciones profesionales y construcciones identitarias de estos estudiantes, siendo este el discurso dominante en sus reflexiones. Por último, este artículo reflexiona sobre la importancia del institucional currículum en el acompañamiento de las trayectorias formativas de futuros profesores de matemática.

\section{METODOLOGÍA}

\section{Enfoque y diseño}

Este estudio forma parte de una investigación doctoral que indaga la construcción de la identidad profesional en contextos de participación y aprendizaje en comunidades de práctica. Con el fin de responder a los objetivos de la investigación 
planteada, el estudio considera una óptica paradigmática interpretativa (Cornejo et al., 2012) y un enfoque biográfico-narrativo (Denzin y Lincoln, 2003; Bolívar y Domingo, 2006) con apoyo de datos estadísticos descriptivos. Tales decisiones buscan enfatizar las cualidades, procesos y motivaciones acentuadas por la naturaleza de la construcción de la identidad del futuro profesor, explorando las dinámicas de esas situaciones concretas a través de la propia experiencia, la percepción y el relato que de ella hacen los futuros docentes de matemáticas que participaron de este estudio.

\section{Participantes y contexto}

Esta investigación se llevó a cabo en una escuela de formación de profesores de matemática de una universidad pública y regional del centro-sur de Chile. Los datos se recolectaron durante el ciclo lectivo 2016 y los participantes son estudiantes de cuarto año adscritos a un curso de práctica pedagógica $(\mathrm{N}=25)$. Se seleccionó esta asignatura debido a que su contenido y la disposición de los académicos a cargo propiciaron la reflexión sobre los fenómenos relacionados a la construcción identitaria de estos futuros profesores de matemáticas. Los participantes se sitúan en un rango de edad entre los 21 y 31 años, siendo la mediana 22 años. En cuanto a su sexo la distribución es equitativa, con 11 estudiantes del sexo masculino (44\%) y 14 del sexo femenino (56\%).

De acuerdo con el cuestionario de caracterización del futuro profesor de matemática (descrito en el punto 1 de Instrumentos), los participantes del estudio han desarrollado su formación de secundaria principalmente en colegios de dependencia administrativa subvencionada particular (61,5\%), mientras que el resto de los alumnos ha estudiado en colegios municipales (38,5\%). No hay registro de alumnos que cursaran su enseñanza media en colegios particulares. Con respecto a la modalidad de enseñanza, el $34,6 \%$ de los participantes se han formado en especialidades técnico-profesionales y el $65,4 \%$ en modalidades científico-humanistas.

Con respecto a los promedios de enseñanza media, el rango declarado va desde notas de 4,9 a 6,9. Mientras que el promedio obtenido durante la enseñanza media para la asignatura de matemática se encuentra en el rango de 6,0 a 7,0, siendo 6,5 el promedio modal de la muestra. Además, es posible apreciar que 3 alumnos declaran haber obtenido la calificación máxima $(7,0)$ en la asignatura como promedio de enseñanza media. 
En lo referido a las características de los puntajes de ingreso a la formación inicial docente, los estudiantes que ingresaron entre los años 2010 y 2013, poseen puntajes promedios mayores a la media nacional (513 puntos; Fuente: DEMRE). En la Prueba de Selección Universitaria (PSU) el rango de puntajes se sitúa entre 550 y 723 puntos y en la Prueba Obligatoria de Matemática los puntajes varían entre 580 y 677 puntos. Asimismo, se reporta que la Pedagogía en Educación Matemática es la primera preferencia en el $92,3 \%$ de los alumnos.

\section{Instrumentos de recolección de datos}

Por la naturaleza de este tipo de estudio, se desarrollaron dos instrumentos complementarios:

\section{1) Encuesta de caracterización del futuro profesor de matemática}

Este instrumento buscó indagar las particularidades de los participantes. En su composición se integraron aspectos personales demográficos, académicos, sociales, motivacionales, laborales y familiares. La elección de tal composición se apoya sobre estudios como los de Parra (2004), Galaz (2011) y Sylvia et al. (2011), quienes entienden la identidad como un proceso dinámico constituido por historias personales, así como también por referencias y pertinencias colectivas.

\section{2) Biogramas}

Este instrumento persiguió explorar las trayectorias de construcción de la identidad profesional bajo la premisa de que en las sucesivas etapas de la formación docente existen acontecimientos y decisiones que han determinado características identitarias en el futuro profesor de matemáticas. Se entendieron estos biogramas como una estructura general y cronológica del recorrido formativo de un individuo donde convergen acontecimientos de su existencia y desarrollo profesional (Sayago et al., 2008). Para Sandín (2003), estos biogramas adquieren especial relevancia cuando se desea comparar y resaltar eventos significativos, tales como lo son aquellos que llevan a la formación de identidad profesional. En la Figura 1 se presenta un ejemplo del biograma utilizado para este estudio. 


\begin{tabular}{|c|c|c|c|}
\hline $\begin{array}{c}\text { Cronología } \\
\text { (Año, semestre, } \\
\text { estación del año, } \\
\text { etc.) }\end{array}$ & $\begin{array}{c}\text { Hecho } \\
\text { (identificación de } \\
\text { acontecimientos, } \\
\text { sucesos y/o } \\
\text { decisiones y una } \\
\text { breve descripción } \\
\text { de la situación } \\
\text { que ha influido } \\
\text { en ti como futuro } \\
\text { profesor) }\end{array}$ & $\begin{array}{c}\text { Contexto } \\
\text { surge la experiencia } \\
\text { desde lo más } \\
\text { próximo al sujeto } \\
\text { hasta el ambiente } \\
\text { sociocultural del } \\
\text { hecho) }\end{array}$ & $\begin{array}{c}\text { Evaluación } \\
\text { (valoración atribuida } \\
\text { en términos de } \\
\text { escala al hecho } \\
\text { descrito) }\end{array}$ \\
& & \\
\hline
\end{tabular}

Figura 1. Fuente: elaboración propia.

\section{Procedimientos de aplicación}

Los datos se recolectaron en dos etapas para las cuales el primer autor del artículo se presentó a dos sesiones de un curso de práctica pedagógica de cuarto año de la carrera de Profesorado en Matemáticas, con el fin de dar a conocer la investigación y aplicar los instrumentos de recolección de datos.

De esta forma, al inicio de la intervención investigativa y al amparo de las consideraciones éticas para el desarrollo de la investigación con seres humanos, de acuerdo a la Declaración de Helsinki (1964), durante la primera sesión con los participantes se les informó los objetivos y alcances del estudio, y se procedió a la firma de su consentimiento informado. Posterior a ello, durante la misma sesión, se aplicó la encuesta de caracterización del futuro profesor de matemática de manera individual a aquellos alumnos que decidieron participar en el estudio, la cual fue respondida en un tiempo promedio de 45 minutos.

En la segunda sesión de intervención y buscando resguardar la privacidad de las respuestas de los biogramas, se utilizó la plataforma informática institucional (MOODLE), a la que el autor principal tiene acceso, para plantear a los estudiantes la tarea a realizar. Así, los individuos participantes respondieron y enviaron de manera virtual las respuestas del biograma (Figura 1).

Instrumentos y procedimientos de análisis

Para el análisis de la información obtenida en la encuesta de caracterización, se llevó a cabo un análisis estadístico descriptivo, en el cual se calcularon frecuencias, medias y porcentajes a modo de obtener una síntesis general de 
las características personales, sociales, académicas y motivacionales de los participantes. Para esto, se incorporó la información en el software de gestión estadística PASW 21, lo que permitió resumir y presentar la información mediante el uso de tablas, gráficos y figuras según la naturaleza de las variables.

Por otro lado, los biogramas se analizaron a fin de buscar evidencias en los hechos, acontecimientos, contextos y valoraciones que los educandos manifestaron que los han construido identitariamente durante su trayectoria como estudiantes de pedagogía. Para ello, se establecieron etiquetas para cada participante, un pseudónimo para cada entrevistado y un número que refleje el orden considerado en la recopilación de la información. Para la comprensión individual y colectiva del grupo, se recopiló esta información en un biograma general (Sayago et al., 2008) que diera cuenta de los hallazgos. Luego, los datos de este biograma general fueron analizados con ATLAS.ti, un paquete de software diseñado para un análisis cualitativo fino y detallado de grandes volúmenes de datos de texto (hablado y escrito) (Paulus y Lester, 2016). Esta herramienta analítica permitió la navegación, procesamiento y estructuración de la información contenida en el biograma general. De esta forma, se identificaron temas emergentes y recurrentes de interés analítico y se establecieron categorías de análisis. Una vez definidas estas categorías, se seleccionaron los extractos que sirven de ejemplos en este artículo por su representatividad y capacidad para ilustrar los temas emergentes y recurrentes de los hechos, motivaciones y percepciones que constituyen las trayectorias de formación, las cuales, a su vez, reflejan la construcción identitaria de estos futuros profesores de matemática.

\section{Resultados del estudio}

\section{Motivaciones en trayectorias profesionales}

Con respecto a las motivaciones, razones o deseos para ingresar a estudiar Pedagogía en Matemática que se recogieron a través del cuestionario de caracterización, el 100\% de los estudiantes manifestaron al menos una razón, tres estudiantes no declararon un segundo motivo y 12 estudiantes no declaran una tercera razón. Los motivos declarados como primera elección de estos futuros profesores se presentan en la Figura 2. 


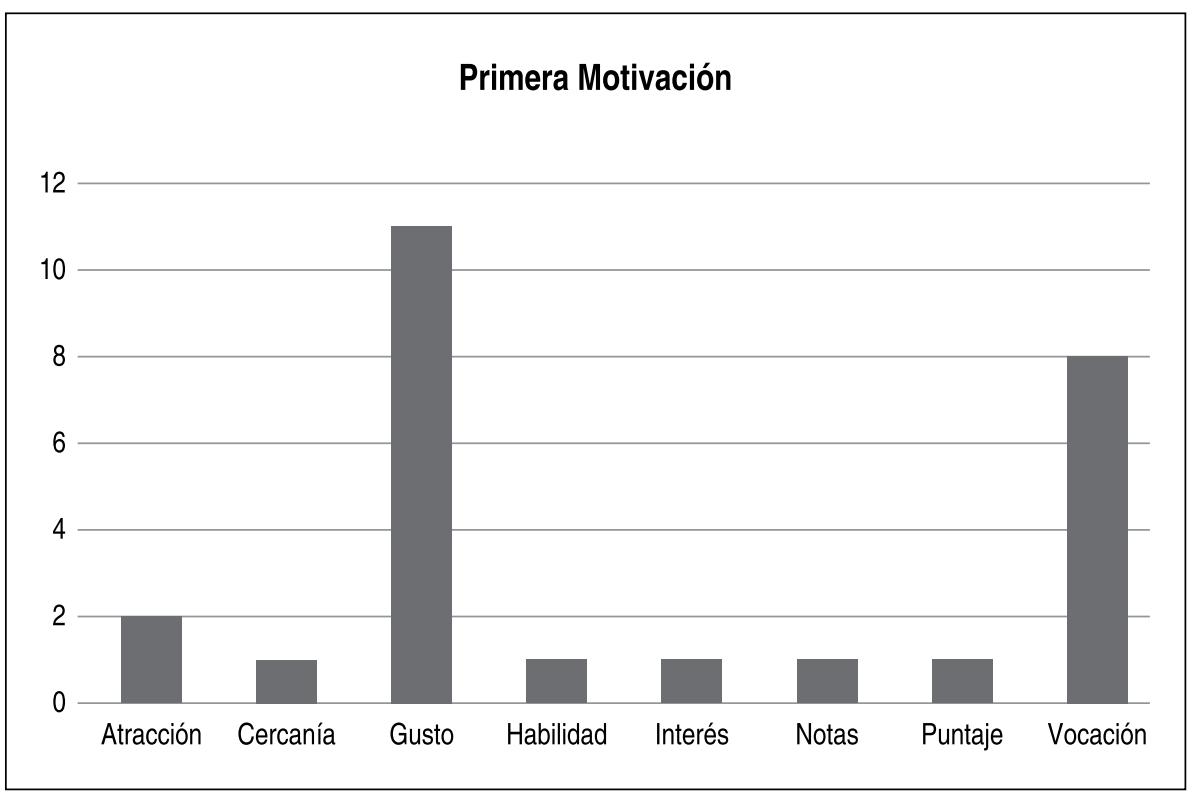

Figura 2. Fuente: elaboración propia.

Como se aprecia, las primeras y mayores frecuencias de motivaciones manifestadas se deben al gusto por las matemáticas $(42,3 \%)$ y a la vocación pedagógica $(30,8 \%)$. Con respecto a las segundas motivaciones para estudiar Pedagogía en Matemática, se observó la vocación docente $(19,2 \%)$, el gusto $(11,5 \%)$ y la habilidad hacia el trabajo matemático $(11,5 \%)$ como respuestas recurrentes. Por su parte, las becas $(11,5 \%)$ y el campo laboral $(11,5 \%)$ surgen como variables que han sostenido sus decisiones para aquellos alumnos que seleccionaron terceras motivaciones.

Al analizar las motivaciones a partir de la variable de género, se aprecia que mientras que la primera motivación de los hombres es el gusto por las matemáticas (72\%), las mujeres declaran que la vocación es su más importante motivación (57\%). Por otra parte, al considerar la segunda motivación hacia la orientación profesional, el $36 \%$ de los hombres incluye a la vocación, mientras que la habilidad $(21 \%)$, las becas (14\%) y el gusto (14\%) son los referentes más frecuentes en las mujeres. Por último, con respecto a las terceras motivaciones, los hombres le asignan más importancia al campo laboral (28,5\%), mientras que las respuestas de las mujeres se dividen uniformemente entre becas, cercanía de la universidad con su lugar de origen, gusto, habilidad y proyección (16,6\%). 
Contexto cronológico, espacio sociocultural y valoración de experiencias de trayectoria profesional

El biograma fue contestado por un total de 25 estudiantes, siendo 11 de ellos hombres y 14 mujeres. Una síntesis general sobre los resultados se expone en las Tablas 1, 2 y 3. La Tabla 1 presenta la distribución por género de la cantidad de hechos y experiencias (correspondiente al segundo ítem de la Figura 1) que los futuros profesores han relatado en los biogramas.

\section{Tabla 1}

Distribución por género de experiencias relatadas en biogramas.

\begin{tabular}{lccc}
\hline & Hombres & Mujeres & TOTALES \\
\hline Experiencias & 77 & 96 & 173 \\
\hline
\end{tabular}

Fuente: elaboración propia.

Los 173 relatos identificados en los biogramas constituyen un corpus de 8021 palabras.

Con respecto a la dimensión cronológica del biograma (correspondiente al primer ítem de la Figura 1), los futuros profesores reflexionaron sobre los momentos o etapas de la vida donde les han surgido inquietudes y situaciones que influyeron de manera positiva, aunque a veces negativa, en su formación profesional. Tales etapas de trayectorias formativas se agruparon de acuerdo a su periodo de escolarización. Los resultados se pueden visualizar en la Tabla 2 a continuación.

\section{Tabla 2}

Contexto cronológico de las manifestaciones.

\begin{tabular}{lccc}
\hline & Hombres & Mujeres & TOTALES \\
\hline Infancia & 0 & 3 & 3 \\
\hline Primaria & 10 & 8 & 18 \\
\hline Secundaria & 30 & 40 & 70 \\
\hline Superior & 41 & 41 & 82 \\
\hline TOTALES & 82 & 93 & 173 \\
\hline
\end{tabular}

Fuente: elaboración propia. 
El período denominado infancia recoge impresiones solo de mujeres (3); esta etapa es previa a la escolarización y al contacto directo con profesores o educadoras. La etapa de primaria, o enseñanza básica, que comprende aproximadamente entre los cinco y los trece años de edad, son recordadas como significantes para ambos sexos. La secundaria y el período de educación superior concentran el mayor conjunto de experiencias positivas, pero también negativas que llevan a construir la decisión de formarse como profesor, así como también el conjunto de hechos, acontecimientos y decisiones que reafirman tal elección. Es posible entonces visualizar que el período de formación inicial docente, con un $44 \%$ de las manifestaciones, es una fuente importante de producción y/o readecuación de su identidad profesional en relación con los demás periodos descritos.

Siguiendo la linealidad del biograma, con respecto al espacio sociocultural donde transcurren tales manifestaciones, es decir, el contexto o espacio físico en el que los estudiantes han evocado sus experiencias (correspondiente al tercer ítem de la Figura 1), estas se sitúan, como se verá en los relatos más abajo, en dos escenarios principalmente: el colegio y la universidad. Estos contextos se relacionan de manera positiva con los resultados del punto previo del biograma que muestra que el contexto de escolarización obligatorio y la formación en educación superior son fundamentales para la toma de decisiones y la construcción de identidad profesional de estos estudiantes.

Por último, con respecto a la valoración de tales experiencias, esto es, el nivel de significancia y de evaluación declarado por los propios participantes a las experiencias y/o acontecimientos relatados en la última columna del biograma, se observan principalmente eventos, situaciones y experiencias fuertemente positivas de influencia hacia una formación en pedagogía en matemáticas.

\section{Tabla 3}

Valoración atribuida a la experiencia.

\begin{tabular}{lccc}
\hline & Hombres & Mujeres & TOTALES \\
\hline Fuerte & 44 & 56 & 100 \\
\hline Media alta & 13 & 7 & 20 \\
\hline Media & 17 & 23 & 40 \\
\hline Media baja & 0 & 1 & 1 \\
\hline Negativa & 4 & 8 & 12 \\
\hline TOTALES & 73 & 90 & 173 \\
\hline
\end{tabular}

Fuente: elaboración propia. 
Como se observa en Tabla 3, solo un 7\% de tales manifestaciones conlleva a hechos que han influido negativamente en la percepción de ser profesor o con respecto a la pedagogía. Se aprecia, además, que son principalmente las mujeres (55\%) quienes asignan rangos altos de influencias a sus experiencias. El $58 \%$ de los relatos realizados por las mujeres han tenido una influencia fuerte en sus decisiones, mientras que en el rango medio de influencias de las experiencias (media baja, media, media alta) la distribución es equitativa entre hombres y mujeres.

Relatos de experiencias formativas: Sobre la influencia de los docentes en la trayectoria formativa

Las experiencias y los acontecimientos que han influido en la toma de decisiones para proseguir estudios de pedagogía en matemática y mantenerse en un proceso de formación docente fueron obtenidos a través de los relatos ofrecidos por los futuros profesores en la segunda columna del biograma. Del análisis de temas emergentes y de sus categorías, se observó que el discurso más dominante con respecto a los hechos y experiencias que han influido en sus trayectorias de formación profesional está predominantemente caracterizado por el rol de los docentes que fueron parte de sus procesos formativos. Como se verá a continuación, los relatos muestran que los profesores de enseñanza básica, media y educación superior se convirtieron en una imagen sobre la cual fijar la atención y en un modelo a seguir en el ámbito profesional, lo cual contribuye no solo a la elección de su carrera universitaria y área de estudios, sino también a la proyección y construcción de su identidad profesional.

El hecho fundamental que me llevó a tomar la decisión de estudiar pedagogía en matemática fue la motivación que me entregaron mis profesores de matemática a lo largo de mis estudios de básica y media. Tuve muy buenos profesores. E18 1

Tuve una clase con un profesor que fue capaz de sorprender y asombrar a cada uno de los estudiantes en esa sala, incluyéndome. E22

El contexto de enseñanza de la matemática de profesores en primaria prevalece en las producciones y son recordadas como experiencias positivas de influencia:

E18 = Estudiante $\mathrm{N}^{\circ} 18$ y así sucesivamente. 
Mi profesor de matemáticas de enseñanza básica tenía especial cuidado en cómo nos enseñaba matemáticas; él lo hacía con pasión, le gustaban mucho las matemáticas. E14

Después de estar ocho años con la profesora [nombre] como profesora jefe, se me fue metiendo el bichito de qué se sentiría el enseñar. E13

Con respecto a las experiencias que involucran a docentes en el período de enseñanza media, los futuros profesores reflexionan sobre el rol de la imagen del profesor como pedagogo y no solo matemático.

En estos años tuve una profesora que me gustó mucho como enseñaba y como trabajaba, [nombre], es una de las personas que influyó sobre mi decisión de estudiar pedagogía. E20

Por su parte, la influencia del docente universitario se da en espacios de formación tanto disciplinar como didáctico-pedagógica.

Durante el segundo semestre del 2015 estuve cursando la asignatura de Álgebra Lineal y el primer semestre del 2016 la de Estructuras Algebraicas, con el profesor [nombre], el cual me ayudó mucho a orientarme para saber a dónde quiero llegar. E20

En sala de clases el profesor [de currículum y evaluación] muestra distintas realidades para la enseñanza de la matemática, motivación personal para llegar a ser como la realidad a la que se debería llegar. E2

De este análisis biográfico de los relatos sobre el rol de los docentes formadores, se identifican, a su vez, tres subcategorías temáticas: actitud de los docentes, apoyo del profesor y la experiencia pedagógica, las cuales se ilustran a continuación.

\section{Tabla 4}

Registros comunicativos sobre el rol de los docentes en trayectorias formativas.

\begin{tabular}{llc}
\hline Categorías & \multicolumn{1}{c}{ Subcategorías } & Registros comunicativos \\
\hline Influencia de los docentes & - Actitud de los docentes. & 57 \\
& - Apoyo del profesor. & \\
& - La experiencia pedagógica. & \\
\hline
\end{tabular}

Fuente: elaboración propia. 


\section{Actitud del docente}

Para Gómez-Chacón (2000), la actitud es posible interpretarla como una predisposición evaluativa que determina comportamientos e influye sobre las intenciones personales. Estas actitudes han sido percibidas en sus diferentes dimensiones por parte de los futuros profesores, convirtiéndose para ellos un referente profesional.

Llegó un nuevo profesor a la escuela; él fue quien logró que a mí me gustara la matemática. E24

Cuando la profesora empezó a enseñar las divisiones se me hacía mucho más fácil resolverlas que a mis compañeros, llamándome así la atención en primera instancia las matemáticas, algo que antes no había pasado. E25

El profesor [nombre] en ese tiempo fue la persona que con sus conocimientos y paciencia me fomentó el gusto por las matemáticas. E9

Cuando el profesor hace evidente sus actitudes hacia la matemática y su proceso de enseñanza y aprendizaje desde la dimensión cognitiva en la institución escolar (Padrón, 2008) esto conlleva a la adaptabilidad en los escenarios de desempeño y se favorece la decisión de actuar de manera preferencial hacia el objeto de enseñanza. Por su parte, la recepción del estudiante de tal actitud es valorada de acuerdo con los resultados de aprendizaje obtenidos.

Estaba en segundo medio, era mi primer día de clases, empezaba con matemáticas y llegó un profesor de avanzada edad. Desde el momento en que habló fue impresionante como se le veía en el rostro su vocación, a pesar de no estar apegado a la tecnología. E15

Como han planteado diversos estudios, en la clase de matemática no solo se producen transferencias de sabes disciplinares, sino también afectivos y actitudinales por parte del docente. Chaves et al. (2008) muestran el esquema de funcionamiento de los componentes afectivos y sus reacciones en los estudiantes. 


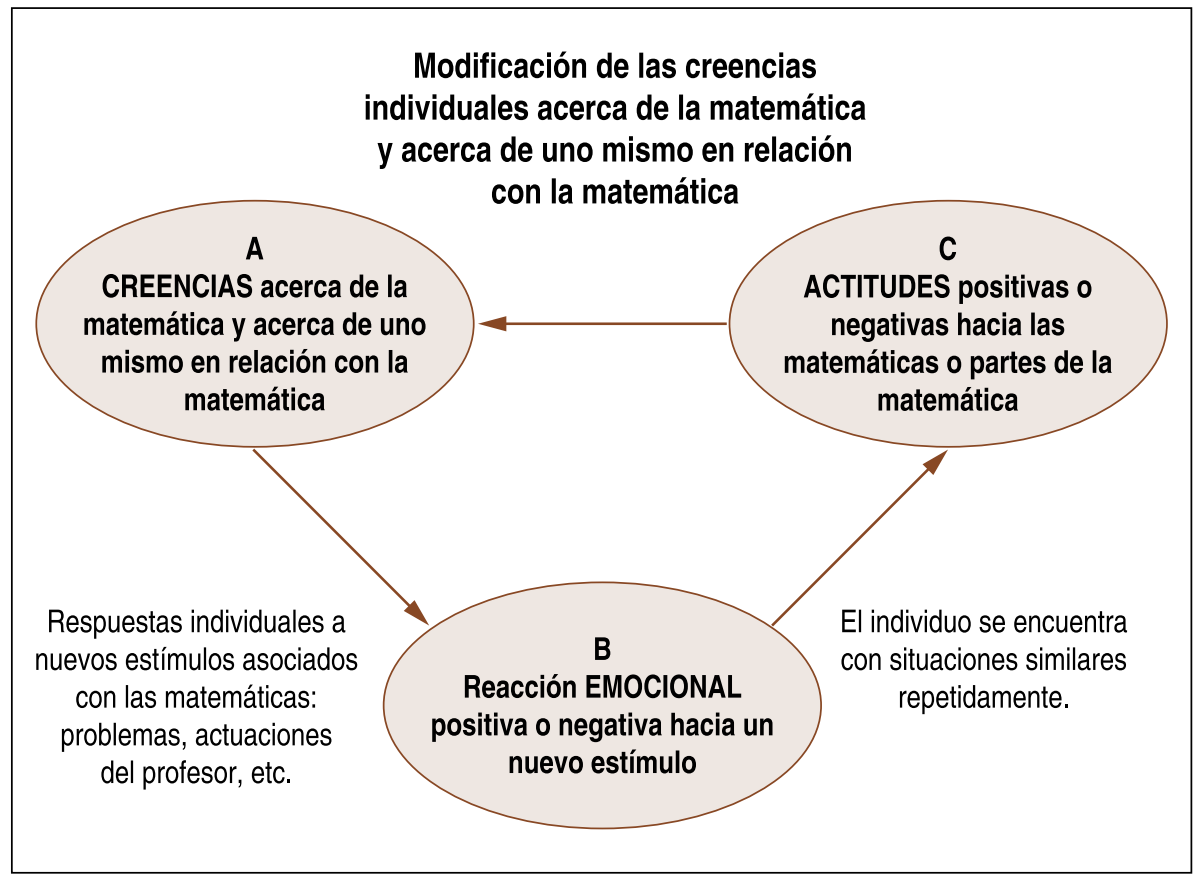

Figura 3. Dimensión afectiva en la enseñanza y aprendizaje de la matemática. Fuente: Chaves et al., (2008).

Tales reacciones planteadas por los autores (Chaves et al., 2008) se muestran en los relatos y van reafirmando un camino de acercamiento hacia la matemática y hacia el docente.

A principio de año llegó una profesora de matemáticas y biología que nos hizo ir descubriendo las matemáticas, lo que me dejó más encantado con la matemática. E24

Mi profesor de matemáticas de enseñanza básica tenía especial cuidado en cómo nos enseñaba matemáticas; él lo hacía con pasión, le gustaban mucho las matemáticas. E25

Aunque la mayoría de la escuela lo consideraba serio y enojón, conmigo se llevaba muy bien porque me veía interesada en sus clases y siempre le estaba haciendo consultas. E14 
De esta forma, los biogramas evidencian la presencia de componentes afectivos en la actitud de los profesores encargados de formar a estos futuros profesores. A partir de los hechos y experiencias, las actitudes para con la enseñanza de la matemática han permeado fuertemente una trayectoria hacia la docencia de la disciplina.

\section{Apoyo del profesor}

Otra subcategoría que emergió sobre el rol de los profesores formadores fue aquella concerniente al apoyo brindado por ellos durante los años de escolarización. Los estudiantes asocian este apoyo del docente en términos de su vocación de servicio, con base en aprendizajes obtenidos producto de su formación y de la dedicación brindada.

Mi profesora de matemática me inspiraba a ser profesora. Me gustaba 10 dedicaba que era en cada clase y el apoyo que me daba. E3

Ya decidido, tenía que empezar a prepararme para la PSU, tema difícil, ya que no tomé preu [pre-universitario, curso de preparación para rendir la PSU] y creo que el apoyo, o la enseñanza generada por los profesores que tuve como el profesor [nombre] y el profesor [nombre], quienes fueron buenos profesores y de quienes aprendí mucha matemática. E20

Cuando cursaba $2^{\circ}$ año medio en el liceo de [localidad] se presenta un profesor, el cual había egresado recientemente de la carrera. En él vi una vocación tan especial que en todos los años de mi educación no lo había visto. Él me ayudó mucho. E21

Otras veces el apoyo ocurre fuera de las esferas formativas establecidas. Las orientaciones de los docentes apoyan decisiones de desarrollo personal y profesional que movilizan la construcción identitaria.

Ese profesor me ayudó dándome cuenta de mi rol, que tendré como futura docente, y me inspiró en seguir estudiando y siempre dar lo mejor de mí. $\mathrm{E} 1$

Me encontré con mi antigua profesora y ella me dio ánimo. E3 
Los relatos de apoyo recibidos de parte de los docentes muestran una alta valoración en lo concerniente a su desarrollo profesional, especialmente como bases que sustentan ideales profesionales. La interacción como soporte que se percibe entre profesores y estudiantes están cargadas de empatías (Hargreaves, 2000) que provocan el situarse al nivel de los sentimientos, desde donde se posiciona el evento identitario.

\section{Experiencia pedagógica}

Con respecto a la tercera y última subcategoría identificada, relacionada al rol del docente formador, la literatura (Vaillant, 2016; Burns y Luque, 2014) muestra que cada vez surge con más fuerza el hecho de que el grado motivacional de un profesor que asume como principales funciones el ayudar, orientar y guiar a sus alumnos está directamente relacionado al uso de metodologías y técnicas que llaman al aprendizaje significativo y despiertan el interés y la participación activa de los alumnos. En este sentido, se encontró que la mayoría de los futuros docentes que participaron en la investigación sustenta sus decisiones formativas en la imagen pedagógica proyectada por sus profesores durante los años de estudio, focalizando sus relatos en las experiencias de las pedagogías y didáctica utilizada por sus formadores. Este conjunto de experiencias ha influido, tanto en el entender disciplinar, como también en la concepción de la importancia de la pedagogía.

A través de diversas actividades propuestas por la profesora para el aprendizaje del eje geométrico, logré observar el interés que creaban en mí estas. E12

Estaba en segundo medio, era mi primer día de clases, empezaba con matemáticas y llegó un profesor de avanzada edad... siempre mostraba metodologías muy cercanas a la realidad que nos permitían comprender de mejor forma las matemática. E15

Con la llegada de un nuevo profesor de matemática que traía consigo una nueva metodología de enseñanza, aumentó más mi interés por las matemáticas. E23

Como aquí se muestra, los relatos hablan de prácticas pedagógicas que aumentan el interés, la comprensión y la valoración hacia la matemática. En los 
relatos también se evidencia la influencia de formación pedagógica recibida en los estudios universitarios, pues se adoptan posiciones didácticas para entender la relación profesor-estudiante.

...su explicación, aunque daba sueño, era la única explicación de matemática que podía entender sin factores externos. E17

Al asistir a clases de preuniversitario de mi liceo, me gustaba la "nueva matemática" que me mostró la profesora, la forma en que lo hacía, sus métodos, y lo fácil que se me hacía, y siempre me preguntaba ¿Si ella puede, por qué yo no?. E1

Como se aprecia, el contacto con el educador en los diferentes niveles formativos constituye una base de buenas experiencias que marcan un proceso profesional de formación. La reducción de la información obtenida en subcategorías de análisis ha permitido indagar en profundidad los hechos y experiencias que hicieron de estos profesores referentes disciplinares y pedagógicos durante los procesos de las trayectorias formativas de estos futuros docentes de matemática.

\section{DISCUSIÓN Y CONCLUSIONES}

Al indagar sobre las motivaciones que llevaron a este grupo de futuros profesores a elegir la pedagogía en matemática como su área de desarrollo profesional, estos nos muestran que el gusto por el saber disciplinar y la vocación por la pedagogía constituyen las razones mejores valoradas y más frecuentes por las cuales se encuentran transitando este camino profesional. Por otra parte, cuando los futuros profesores reflexionan sobre los acontecimientos, experiencias y orientaciones formativas, se encuentra que los docentes de matemática que les impartieron clase a lo largo de su educación formal despertaron y/o reafirmado su vocación por la enseñanza de las matemáticas, a la vez que fomentaron el gusto por el saber disciplinar.

Se visualiza entonces que el futuro profesor transita experiencias caracterizadas por diferentes estilos y personalidades de maestros formadores, y, en ese devenir, percibe diversos elementos de estas experiencias formativas relacionadas con sus profesores referentes como fuentes de motivación, que conjuga altos niveles de preferencia por las metodologías y didácticas utilizadas y que sirve de inspiración para el establecimiento de metas e ideales profesionales que lo ayudan a definir 
qué tipo de profesional desea ser. Tales referencias son percibidas en términos de, por ejemplo, pasión por la enseñanza, motivación entregada en las clases, la capacidad de asombrar y encantar, y el gusto por la disciplina, percepciones que se han agrupado de acuerdo a la valoración por: a) la actitud de los docentes, b) el apoyo entregado por el profesor en el proceso de aprendizaje, y c) la experiencia pedagógica. Así, los relatos dan evidencia de que esta valoración por las experiencias con profesores referentes se vincula de manera muy positiva con el desarrollo de las trayectorias formativas de los futuros pedagogos.

El preponderante rol que juega el profesor de matemática en servicio de las trayectorias formativas de futuros pedagogos muestra que el docente ha trascendido al aula, contribuyendo no solo a la elección de la carrera profesional, sino también a la formación de identidad del futuro profesional. Esto plantea la interrogante sobre cómo la formación inicial docente atiende e incorpora estos aspectos altamente valorados de las trayectorias formativas, así como en las orientaciones y motivaciones identitarias acumuladas durante la trayectoria formativa en su visión y misión pedagógica. En este sentido, Cols (2008: 3) explica que estos aspectos de las trayectorias formativas deben estar plasmados en el texto curricular, pues es la institución quien mediante "un currículum que, en tanto proyecto, anticipa un sentido y define un trayecto deseado". Sin embargo, ¿son estas valoraciones y orientaciones identitarias de los futuros profesores evidentes en el actual sistema de formación inicial de Chile?

Desafortunadamente, desde la formación docente, el acento sigue estando, muy frecuentemente, en los contenidos curriculares, aquellos que el docente necesita adquirir, para luego impartir el conocimiento disciplinar a los educandos. Sin embargo, los relatos de los futuros profesores que participaron en este estudio muestran que su énfasis, es decir las experiencias que han marcado el rumbo de sus trayectorias formativas e identitarias, está puesto en aquellos actores sociales que con sus actitudes hacia la disciplina, con su orientación y apoyo pedagógico han guiado los pasos a seguir desde los comienzos de su educación formal.

Los docentes, como agentes formativos, deben entonces ocupar un rol más central en la visión institucional de la pedagogía y el currículum. En este sentido, se propone poner un mayor énfasis en la formación de competencias blandas, que permitan a los educandos reflexionar y desarrollar aspectos de su práctica docente que favorezcan las relaciones interpersonales con el alumnado, apoyando sus motivaciones vocacionales y el desarrollo de metas e ideales profesionales (Fernández, 2006). Además, pensamos que es necesario realizar 
investigaciones futuras que pongan de manifiesto las experiencias obtenidas desde las trayectorias formativas, pues se cree que son aspectos fundamentales en la construcción identitaria de los futuros docentes y de su práctica profesional. Esto puede servir como base de actualización de la visión y misión de la formación docente universitaria y del empoderamiento del futuro profesor, al buscar conjugar los intereses formativos y el conocimiento disciplinar.

\section{AGRADECIMIENTOS}

Rodrigo Panes Chavarría agradece a CONICYT-PFCHA/Doctorado Nacional/2017-21170711 por el apoyo brindado en el desarrollo de su tesis y Grupo de Investigación en Educación y Educación Matemática Gl171323 EF de la Universidad del Bío-Bío. Los autores también desean agradecer al doctor Alberto Galaz por sus valiosos comentarios en los informes de avance de tesis, los cuales contribuyeron a mejorar la reflexión sobre el tema de estudio de este artículo.

\section{REFERENCIAS BIBLIOGRÁFICAS}

ÁVALOS, B. (2014). La formación inicial docente en Chile: Tensiones entre políticas de apoyo y control. Estudios Pedagógicos, 40(ESPECIAL), 11-28.

AVENDAÑO BRAVO, C. Y GONZÁLEZ URRUTIA, R. (2012). Motivos para ingresar a las carreras de Pedagogía de los estudiantes de primer año de la Universidad de Concepción. Estudios Pedagógicos, 38(2), 21-33.

BEDACARRATX, V. (2012). Socialización profesional en el contexto del nuevo milenio: acerca de la tramitación del malestar docente en los trayectos de práctica pre-profesional. Revista Mexicana de Investigación Educativa, 17(54), 903-926.

BEIJAARD, D., MEIJER, P. C. Y VERLOOP, N. (2004). Reconsidering research on teachers' professional identity. Teaching and Teacher Education, 20(2), 107-128.

BOLÍVAR, A., FERNÁNDEZ, M. Y MOLINA, E. (2005). Investigar la identidad profesional del profesorado: Una triangulación secuencial. Forum: Qualitative Social Research, 6(1), 1-26. 
BOLÍVAR, A. Y DOMINGO, J. (2006). La investigación biográfica y narrativa en Iberoamérica: Campos de desarrollo y estado actual. Forum: Qualitative Social Research, 7(4), 1-43.

BOURDIEU, P. (1997). Razones prácticas. Sobre la teoría de la acción. Barcelona: Anagrama.

BURNS, B. Y LUQUE, J. (2014). Profesores excelentes. Cómo mejorar el aprendizaje en América Latina y el Caribe. Washington D.C.: BID.

CHAVES, E., CASTILLO, M. Y GAMBOA, R. (2008). Creencias de los estudiantes en los procesos de aprendizaje de las matemáticas. Cuadernos de Investigación y Formación en Educación Matemática, 3(4), 29-44.

COBB, P., GRESALFI, M. Y HODGE, L. L. (2009). An interpretive scheme for analyzing the identities that students develop in mathematics classrooms. Journal for Research in Mathematics Education, 40-68.

COLS, E. (2008). La formación docente inicial como trayectoria. Documento de trabajo realizado en el marco del Ciclo de Desarrollo Profesional de Directores. Buenos Aires: Instituto Nacional de Formación Docente. Disponible en Centro de Documentación Virtual: http://cedoc. infd.edu.ar. Accedido el 27-01-18.

CONTRERAS-SANZANA, G. Y VILLALOBOS-CLAVERÍA, A. (2010). La formación de profesores en Chile: una mirada a la profesionalización docente. Educación y Educadores, 13(3), 397-417.

CORNEJO, J.; SANHUEZA, S. Y RIOSECO, M. (2012). Orientaciones para la elaboración de tesis, seminarios y paper académicos. Chile: Universidad Católica del Maule.

DENZIN, N. Y LINCOLN, Y. (2003) (Eds.). The landscape of qualitative research: Theories and issues. London: Sage.

EVETTS, J. (2003). The construction of professionalism in new and existing occupational contexts: Promoting and facilitating occupational change. International Journal of Sociology and Social Policy, 23(4/5), 22-35. 
FERNÁNDEZ, J. (2006). El prácticum por competencias: implicaciones metodológico-organizativas y evaluativas. Bordón, 58(3), 121-139.

FERNÁNDEZ, M. (2016). Educación física y formación docente: Impacto de las prácticas pedagógicas en las trayectorias formativas universitarias. InterCambios: Dilemas y Transiciones de la Educación Superior, 3(2), 105-111.

GALAZ, A. (2011). El profesor y su identidad profesional: ¿facilitadores u obstáculos del cambio educativo? Estudios Pedagógicos, 37(2), 89-107.

GÓMEZ-CHACÓN, I. (2000). Matemática Emocional: Los afectos en el aprendizaje matemático. Madrid: Narcea, S. A. Ediciones.

GROOTENBOER, P. (2006). Mathematics educators: Identity, beliefs, roles and ethical dilemmas. Identities, Cultures and Learning spaces, 1, 270-277.

GYSLING, J. (1992). Profesores: un análisis de su identidad social. Santiago: Editorial Universidad Diego Portales.

HABERMAS, J. (1989). Identidades nacionales y postnacionales. Madrid: Tecnos.

HARGREAVES, A. (2000). Mixed emotions: Teachers' perceptions of their interactions with students. Teaching and Teacher Education, 16(8), 811-826.

HUSU, J. (2007) Constructing Teaching as Identity Practice Linking Teachers Individual minds to their School Communities. En Zellermayer, M. y Munthe, E. (Eds.), Teachers Learning in Communities, pp. 45-60. Rotterdam: Sense Publishers.

Lave, J. (1991). Situating learning in communities of practice. Perspectives on Socially Shared Cognition, 2, 63-82.

Lazzaro-Salazar, M. (2013). Diving into the depths of identity construction and motivation of a foreign language learner. Argentinian Journal of Applied Linguistics, 1,1:6-23.

Lazzaro-Salazar, M. (2017). Social-constructionism. En Bernadette Vine (Ed.), The Routledge Handbook of Language in the Workplace (capítulo 8). London: Routledge. 
NASIR, N. (2007). Identity, Goals, and Learning: The Case of Basketball Mathematics. En Nasir, N. y Cobb, P. (Eds.), Improving Access to Mathematics: Diversity and Equity in the Classroom, pp. 132-145. New York: Teachers College Press.

NAVARRETE-CAZALES, Z. (2015). ¿Otra vez la identidad?: Un concepto necesario pero imposible. Revista Mexicana de Investigación Educativa, 20(65), 461-479.

PADRÓN, O. (2008). Actitudes hacia la matemática. Sapiens. Revista Universitaria de Investigación, 9(1), 237-256.

PARRA, M. (2004). La construcción de la identidad profesional del docente: un desafío permanente. Enfoques Educacionales, 29-49.

PAULUS, T. Y LESTER, J. (2016). ATLAS.ti for conversation and discourse analysis studies. International Journal of Social Research Methodology, 19(4), 405-428.

PEDRAJA-REJAS, L., ARANEDA-GUIRRIMAN, C., RODRÍGUEZ-PONCE, E. Y RODRÍGUEZ-PONCE, J. (2012). Calidad en la formación inicial docente: evidencia empírica en las universidades chilenas. Formación Universitaria, 5(4), 15-26.

SANDíN, M. (2003). Investigación cualitativa en educación. Fundamentos y tradiciones. España: McGraw-Hill.

SAYAGO, Z., CHACÓN, M. Y ROJAS, M. (2008). Construcción de la identidad profesional docente en estudiantes universitarios. Educere, 12(42), 551-561.

SFARD, A. Y PRUSAK, A. (2005). Telling identities: In search of an analytic tool for investigating learning as a culturally shaped activity. Educational Researcher, $34(4), 14-22$.

CHONG, S., LOW, E. L., \& GOH, K. C. (2011). Emerging Professional Teacher Identity of Pre-Service Teachers. Australian Journal of Teacher Education, 36(8), 50-64. 
TATTO, M., PECK, R., SCHWILLE, J., BANKOV, K., SENK, S. L., RODRÍGUEZ, M. Y ROWLEY, G. (2012). Policy, Practice, and Readiness to Teach Primary and Secondary Mathematics in 17 Countries: Findings from the IEA Teacher Education and Development Study in Mathematics (TEDS-MM). International Association for the Evaluation of Educational Achievement. Herengracht 487, Amsterdam, 1017 BT, The Netherlands.

UNESCO. (2013). Situación Educativa de América Latina y el Caribe: Hacia la educación de calidad para todos al 2015. Santiago de Chile: OREALC/UNESCO Santiago.

VAILLANT, D. (2010). La identidad docente. La importancia del profesorado. Revista Novedades Educativas, 234, 4-11.

VAILLANT, D. (2016). Algunos marcos referenciales en la evaluación del desempeño docente. Revista Iberoamericana de Evaluación Educativa, 1(2), 8-22.

VERA NORIEGA, J. Y VALENZUELA MEDINA, J. (2012). El concepto de identidad como recurso para el estudio de transiciones. Psicologia \& Sociedade, 24(2): 272282.

WENGER, E. (1998). Communities of practice: Learning, meaning, and identity. Cambridge, UK: Cambridge University Press.

WENGER, E. (2010). Communities of practice and social learning systems: the career of a concept. En Blackmore, C. (Ed.), Social learning systems and communities of practice, pp. 179-198. London: Springer. 